\title{
Abundance studies of sdB stars using UV echelle HST/STIS spectroscopy ${ }^{\star}$
}

\author{
S. J. O’Toole ${ }^{\star}$ and U. Heber
}

\begin{abstract}
Dr Remeis-Sternwarte, Astronomisches Institut der Universität Erlangen-Nürnberg, Sternwartstr. 7, Bamberg 96049, Germany e-mail: otoole@aao.gov.au
\end{abstract}

Received 29 July 2005 / Accepted 20 February 2006

\section{ABSTRACT}

\begin{abstract}
Aims. We test the hypothesis that the pulsations in sdB stars are correlated with the surface abundances of iron-group elements. Any correlation might explain why, when given two spectroscopically similar stars, one will pulsate while the other will not.

Methods. We have obtained high-resolution ultraviolet spectra two pulsating and three non-pulsating sdB stars using the Space Telescope Imaging Spectrograph onboard the Hubble Space Telescope. We determined abundances for 25 elements including the iron group and even heavier elements such as tin and lead using LTE curve-of-growth and spectrum synthesis techniques.

Results. We find no clear correlation between pulsations and metal abundances, and we comment on the resulting implications, including whether it is possible to determine the difference between a pulsating and a non-pulsating sdB spectroscopically. In addition to the main goal of our observations, we have also investigated the effect of supersolar metallicity on fundamental parameter determination, possible trends with iron abundance, and the hypothesis that weak winds may be selectively removing elements from the stellar envelopes. These effects provide challenges to stellar atmosphere modelling and diffusion models for sdB stars.
\end{abstract}

Key words. stars: subdwarfs - stars: abundances - stars: oscillations

\section{Introduction}

The subdwarf B (sdB) stars are core helium-burning objects with envelopes that are too thin to sustain nuclear burning (e.g. Heber 1986). They can be identified with models of Extreme Horizontal Branch (EHB) stars; in other words, they have masses $\sim 0.5 M_{\odot}$ and will evolve directly to the white dwarf cooling curve, bypassing the Asymptotic Giant Branch. While their future evolution seems secure, the formation of these stars is uncertain. In recent years radial velocity surveys have shown that a large fraction of sdBs are in short-period binary systems (Maxted et al. 2001), it appears certain that binary interaction plays a significant role, however many objects are apparently single. A binary population synthesis study by Han et al. (2003) has found that three channels can give rise to the observed characteristics of sdBs: one or two phases of common envelope evolution; stable Roche lobe overflow; and the merger of two helium-core white dwarfs. The latter scenario could explain the population of single stars.

The possibility of pulsations in sdB stars was theoretically predicted by Charpinet et al. (1996) at around the same time they were observed by Kilkenny et al. (1997). The more than 30 known pulsators (officially known as V361 Hya stars) have $T_{\text {eff }}=29000-35000 \mathrm{~K}$ and $\log g=5.2-6.0$, periods of 1-10 min and amplitudes less than $60 \mathrm{mmag}$ (see Kilkenny 2002, for a review). The driving mechanism of the oscillations is believed to be related to the ionisation of iron and other heavy

* Based on observations made with the NASA/ESA Hubble Space Telescope, which is operated by the Association of Universities for Research in Astronomy, Inc., under NASA contract NAS 5-26555. These observations are associated with program \#8635.

$\star \star$ Present address: Anglo-Australian Observatory, PO Box 296, Epping NSW 1710, Australia. elements at the base of the photosphere (Charpinet et al. 1997). As is the case for other types of pulsators (e.g. the PG 1159 stars, Quirion et al. 2004) there is an overlap in the $\left(T_{\text {eff }}, \log g\right)$ plane between pulsators and non-pulsators (Koen et al. 1999). Diffusion calculations by Charpinet et al. (1997) suggest that the surface iron abundance of pulsators should be higher than that of non-pulsators, however studies by Edelmann et al. (2006), Heber \& Edelmann (2004) and Heber et al. (2000, hereafter HRW) find that iron has approximately solar abundance in most sdBs.

For this reason we set out to determine if any correlation exists between surface abundances of iron-group elements for pulsators and non-pulsators. Since elements such as nickel, manganese and chromium are not normally accessible through ground-based optical spectra, it was necessary to acquire highresolution UV echelle spectra with the Space Telescope Imaging Spectrograph onboard the Hubble Space Telescope (HST/STIS). If it is not possible to separate groups based on abundances, perhaps differing mass-loss rates contribute significantly, as suggested by Fontaine \& Chayer (1997). Regardless of this, our abundance measurements will be extremely useful for testing diffusion theory. Previous studies of sdBs using UV spectra from the International Ultraviolet Explorer (IUE) suffered from mediocre $\mathrm{S} / \mathrm{N}$ as well as from poor, or a lack of, atomic data; this was especially the case for the iron group (e.g. Baschek et al. 1982a,b).

The two pulsators we have chosen to observe are Feige 48 (Koen et al. 1998), and PG 1219+534 (Koen et al. 1999). Quantitative analysis of the first two objects using optical spectra was carried out by HRW and the latter by Heber et al. (1999). As comparison objects we have chosen Feige 66 and CD $-24^{\circ} 731$ (alias SB 707); a high-resolution IUE spectrum of the former was analysed by Baschek et al. (1982a). A very highresolution spectrum of the $\mathrm{SdB} C \mathrm{CPD}-64^{\circ} 481$ was found in the 
Table 1. Observations of six sdB stars using HST/STIS. Note that CPD $-64^{\circ} 481$ was observed only in the FUV and using the E140H grating.

\begin{tabular}{lcccccccccc}
\hline \hline Target & $\begin{array}{c}\alpha \\
(2000)\end{array}$ & $\begin{array}{c}\delta \\
(2000)\end{array}$ & $\begin{array}{c}V \\
(\mathrm{mag})\end{array}$ & $\begin{array}{c}T_{\text {eff }} \\
(\mathrm{K})\end{array}$ & $\log g$ & $\log (\mathrm{He} / \mathrm{H})$ & Ref. & $\begin{array}{c}\text { Pulsator? } \\
T_{\text {exp }}^{\text {NUV }} \\
(\mathrm{s})\end{array}$ & $\begin{array}{c}T_{\text {exp }}^{\text {FUV }} \\
(\mathrm{s})\end{array}$ \\
\hline PG 1219+534 & 122129.1 & +530437 & 13.2 & 33500 & 5.87 & -1.6 & 4 & yes & $3100 / 3100$ & $2160 / 3100$ \\
Feige 48 & 114714.5 & +611532 & 13.5 & 29500 & 5.54 & -2.9 & 2 & yes & $3500 / 3511$ & $3600 / 3600$ \\
CPD -64 481 & 054759.3 & -642303 & 11.3 & 27500 & 5.60 & -2.5 & 3 & no & -14 & 1440 \\
Feige 66 & 123723.5 & +250360 & 10.6 & 34500 & 5.83 & -1.6 & 4 & no & 824 & 875 \\
CD -24 731 & 014348.6 & -240510 & 11.0 & 35400 & 5.90 & -2.9 & 4 & no & 800 & 878 \\
\hline
\end{tabular}

References: (1) Heber et al. (1999); (2) Heber et al. (2000); (3) O’ Toole et al. (2005); (4) this work.

HST archive; since its temperature is close to that of Feige 48, and it is not pulsating (Koen, private communication), we decided to use it as a comparison star to Feige 48. Note that these two stars, along with $\mathrm{CD}-24^{\circ} 731$, are in close binaries. Feige 48 and CPD $-64^{\circ} 481$ have periods of $0.376 \mathrm{~d}$ and $0.2772 \mathrm{~d}$ respectively, while CD $-24^{\circ} 731$ has a period of $5.85 \mathrm{~d}$ (O'Toole et al. 2004; Edelmann et al. 2005). The companions of Feige 48 and $\mathrm{CD}-24^{\circ} 731$ are both most likely white dwarfs, while the nature of the companion of CPD $-64^{\circ} 481$ is uncertain.

In this paper we present a detailed abundance analysis of each of these objects based on UV echelle spectra obtained using $H S T / S T I S$. We discuss the possible correlation between iron-group abundances and pulsation, the abundances of heavy elements in the context of radiative acceleration and the trends discussed by O'Toole (2004), and a solution to the temperature discrepancy seen between Balmer line fitting and helium ionisation equilibrium.

\section{Observations}

Observations were made using the Space Telescope Imaging Spectrograph (STIS) onboard the Hubble Space Telescope $(H S T)$. We observed five stars, three pulsators and two nonpulsators for comparison. One of the non-pulsators, Feige 66 is a spectroscopic twin of one of the pulsator PG $1219+534$. Details of the observations are shown in Table 1. The stars were observed in the near- and far-UV (NUV and FUV) using the medium resolution echelle E140M and E230M grisms. The NUV spectra each have a central wavelength of $1978 \AA$, covering the wavelength range $1700-2370 \AA$, while the FUV spectra are centered on $1425 \AA$, covering the $1160-1730 \AA$ range. For all spectra a slit width of $0.2^{\prime \prime} \times 0.06^{\prime \prime}$ was used. Each pulsator was observed in time-tag mode, where the arrival times of each photon is recorded; the two non-pulsators were observed in histogram mode. The spectra of Feige 48 have yielded one piece of serendipity - the discovery of velocity variations indicating a binary companion to the star (O'Toole et al. 2004). Additionally, we found very high-resolution spectra of Feige 66 and $\mathrm{CPD}-64^{\circ} 481$, taken using the E140H grism with a $0.2^{\prime \prime} \times 0.2^{\prime \prime}$ slit covering the range $1163-1363 \AA$. All of the spectra are sharp-lined as expected from the low projected rotation velocities seen in sdBs (O'Toole et al. 2004; and HRW).

\section{Synthetic spectra}

In hot subdwarfs, most of the spectral lines due to iron-group elements (V, Cr, Mn, Fe, Co, Ni) are found blueward of $\sim 2300 \AA$, and in many cases continuum definition is difficult at the resolution of our spectra. The methods we used to get around these problems are discussed in more detail in Sect. 4.1.
As input for our spectrum synthesis, we used a metal lineblanketed LTE model atmosphere with solar metalicity and Kurucz' ATLAS6 Opacity Distribution Functions. The spectra were synthesised using Michael Lemke's version of the LINFOR program (originally developed by Holweger, Steffen, and Steenbock at Kiel University). Oscillator strengths were taken from the Kurucz line list, as were damping constants for all metal lines. Only lines that have been observed experimentally were used, since we required the most accurate wavelengths possible. In the case of species heavier than $\mathrm{Zn}$, values were taken from the resonance line lists of Morton (2000, 2003). For the partition functions of Ga III, Ge IV, Sn IV and Pb IV we used these ions' ground state statistical weight, since no published data is available. This is a good approximation at temperatures of $30000-35000 \mathrm{~K}$. The $\mathrm{Cu}$ III atomic data were taken from the tables of Hirata \& Horaguchi (1995). Oscillator strengths for Ti III lines were taken from Raassen \& Uylings (1997). Lines with $\lambda>2000 \AA$ were converted from air to vacuum wavelengths using the formula of Edlén (1966).

\subsection{Atmospheric parameters}

The determination of atmospheric parameters for the two pulsators to be discussed here was presented by HRW (the values are shown in Table 1). We selected Feige 66 and CD $-24^{\circ} 731$ as potential comparison stars for the pulsator PG 1219+534 because they are not known to pulsate and published temperatures and gravities are similar to those of the latter. While PG $1219+534$ has $T_{\text {eff }}=33500 \mathrm{~K}, \log g=5.85$ from Balmer line analysis by HRW, Feige 66 has $T_{\text {eff }}=33400 \mathrm{~K}, \log g=6.2$ (Saffer et al. 1994) and CD $-24^{\circ} 731$ has $T_{\text {eff }}=34000 \mathrm{~K}$, $\log g=6.0$ (Heber et al. 1984b).

For this work we have reanalysed the Keck-HIRES spectrum of PG 1219+534 from HRW. A high resolution (0.1 A) optical spectrum of CD $-24^{\circ} 731$ was kindly provided by M. Altmann and H. Edelmann. It was taken with the FEROS spectrograph at the ESO $2.2 \mathrm{~m}$ telescope. A low resolution $(5 \AA)$ spectrum of Feige 66 taken with the CAFOS spectrograph was provided by M. Altmann. The spectral analysis of both spectra is described below. The parameters for CPD $-64^{\circ} 481$ have already been determined by O'Toole et al. (2005).

\subsection{The hot sdB temperature discrepancy}

As has been noted by HRW, there is a discrepancy between temperatures derived from Balmer line fitting and helium ionisation equilibrium. For PG $1219+534$, the difference in $T_{\text {eff }}$ is $2000 \mathrm{~K}$.

After the initial discovery of strongly supersolar abundances of heavy metals in three of the programme stars (Feige 66, CD $-24^{\circ} 731 \&$ PG 1219+534), we investigated how this affects the determination of the atmospheric parameters. HRW used 


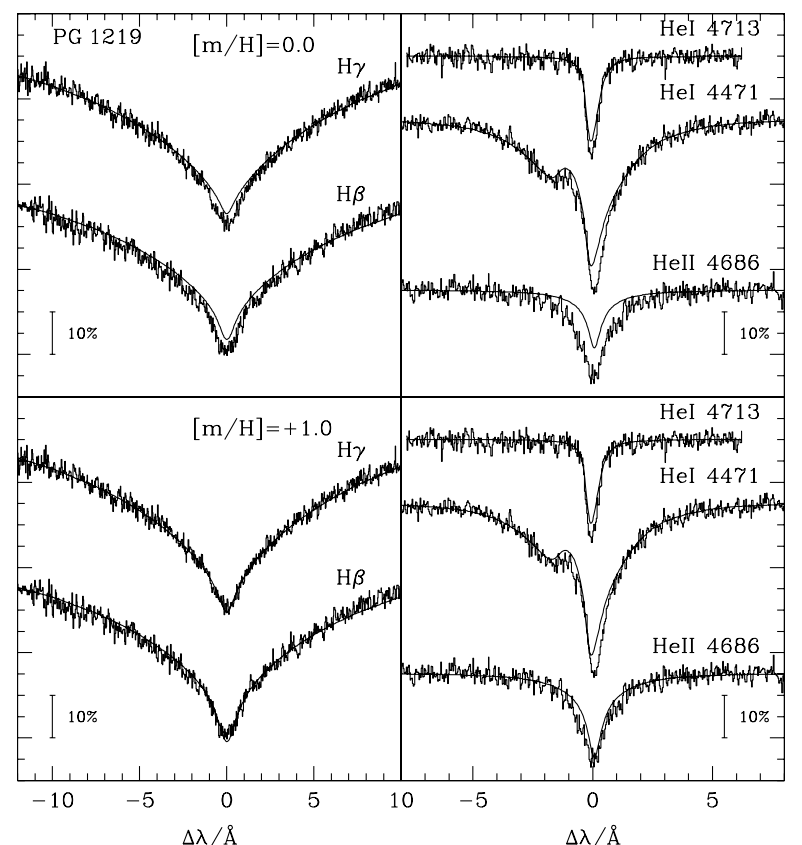

Fig. 1. Line profile fit for PG $1219+534$ using solar metalicity models (top panel) and metal-rich models (10 times solar, bottom panels). The Balmer lines and He II at $4686 \AA$ match simultaneously when using the metal rich models (see text).

metal-line blanketed models with solar metal content. Using the same model atmospheres described above but with metals scaled by a factor of $10([\mathrm{M} / \mathrm{H}]=1.0)$, we have recalculated both our abundances and stellar parameters $\left(T_{\mathrm{eff}}, \log g\right)$. There are currently no opacity distribution functions with higher metalicities. Also, despite the fact that iron itself is show roughly solar abundances for all our targets, in many cases the nickel abundances is 1-2 dex above solar. It is possible that in these cases nickel may become the dominant source of line opacity.

In Fig. 1 we show a fit with these models to the optical spectrum of PG $1219+534$. The Balmer lines and the He II can now be matched simultaneously. The parameters we derive with our metal-enhanced models using all lines are closest (within errors) to those found by fitting the Balmer lines only with a solarmetalicity model. The He I lines agree reasonably well, although the line cores of the strongest lines match poorly. We expect ultimately the solution will be found using opacity sampled models that can allow for enhanced abundances relative to iron.

For CD $-24^{\circ} 731$ we found the same mismatch of the helium ionisation equilibrium as for PG $1219+534$ when solar metalicity models were used (see Fig. 2). Again this problem is remedied by using metal-enriched models. In this case a significantly higher effective temperature was derived from the metalenriched models than from the solar metalicity ones, i.e. $T_{\text {eff }}=$ $35400 \mathrm{~K}$ compared with $T_{\text {eff }}=33800 \mathrm{~K}$. CD $-24^{\circ} 731$ is a rather helium poor star compared to Feige 66 and PG 1219+534, which might explain the different metallicity dependence of the results. In addition, $\mathrm{CD}-24^{\circ} 731$ resides in a close binary system $(P=5.85 \mathrm{~d})$, whereas Feige 66 is not known to be variable (Green, private communication). PG $1219+534$ is also not known to be a radial velocity variable.

The low resolution spectrum of Feige 66 was analysed in the same way with the metal-enriched models and we derived $T_{\text {eff }}=34500 \mathrm{~K}, \log g=5.83, \log (\mathrm{He} / \mathrm{H})=-1.6$. In this case the parameters agree with those found using non-LTE models; however, the He II $4686 \AA$ A line does not agree with observations

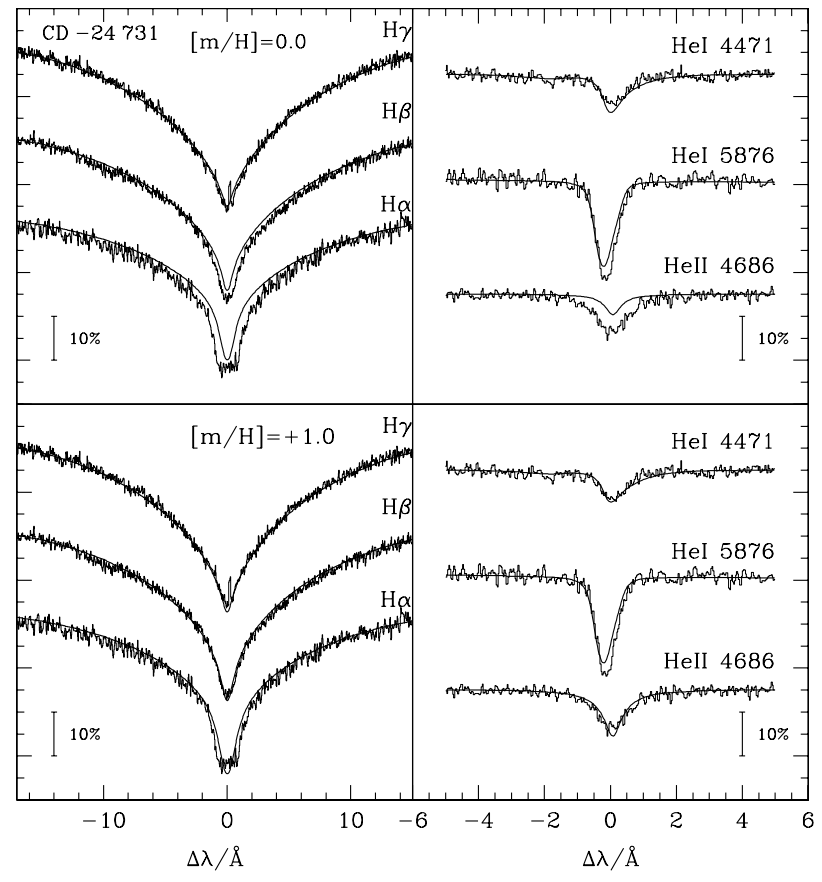

Fig. 2. Same as Fig. 1 but for $\mathrm{CD}-24^{\circ} 731$.

in the enhanced metallicity case, but does in the non-LTE case. A high-resolution spectrum is urgently required to resolve this issue.

The results of the new spectral analyses of PG $1219+534$, CD $-24^{\circ} 731$ and Feige 66 revealed that their surface gravities are identical. The helium abundances of PG 1219+534 and Feige 66 are also identical, whereas helium abundance of $\mathrm{CD}-24^{\circ} 731$ is much lower than for the other two. Their effective temperatures, however, are not identical as suggested by the earlier spectral analyses. Feige 66 and CD $-24^{\circ} 731$ are hotter than PG $1219+534$ by $1000 \mathrm{~K}$ and $1900 \mathrm{~K}$, respectively.

The temperatures we derive here match those determined using metal-free, non-LTE models by HRW. Recently, Charpinet et al. (2005) used TLUSTY and SYNSPEC NLTE models and confirm the NLTE results of HRW for PG $1219+534$ to within $500 \mathrm{~K}$ and 0.04 dex. Since the heavy metal abundances of Feige 48 and CPD $-64^{\circ} 481$ are much closer to solar than those of the former, a reanalysis of these stars is not necessary. The abundances shown in Table 2 are derived from models with these parameters.

\section{Line identification}

As has already been noted, previous studies of high resolution UV spectra of hot subdwarfs have suffered from poor, or a lack of, atomic data. While the current situation is by no means perfect, we have endeavoured to identify as many spectral lines as possible, and then from these we have chosen suitable lines to measure equivalent widths or carry out spectrum fitting. Our method is outlined below, beginning with a brief discussion of the problems encountered with continuum definition.

\subsection{Defining the continuum}

In each of our spectra, the continuum is well-defined in the NUV, especially redward of $2000 \AA$, allowing equivalent widths to be measured. An example of the difference between crowded and well-defined is shown in Fig. 3. There are also some regions below $2000 \AA$ where equivalent width measurements are 

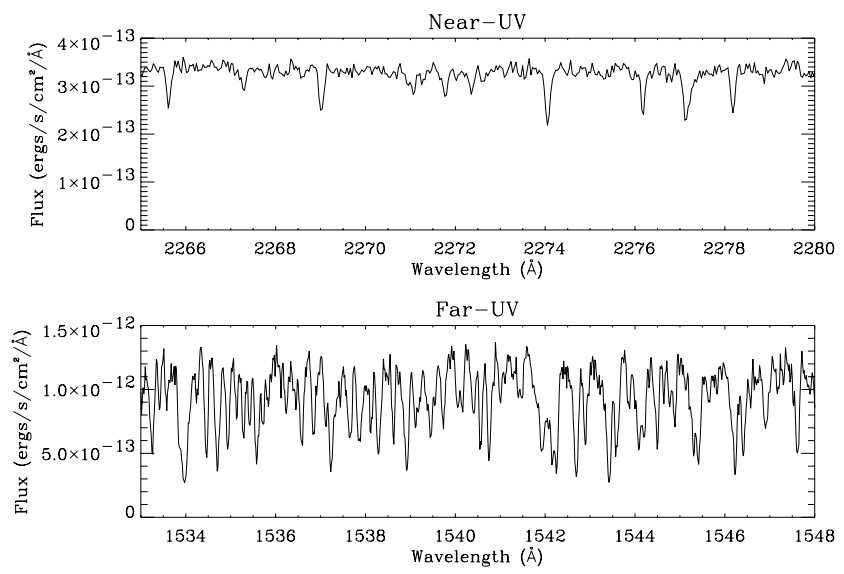

Fig. 3. Comparison of a $15 \AA$ wide section of the near- and far-UV spectrum of PG $1219+534$. In the NUV it is possible to measure the continuum level, while in the FUV it must be estimated. Note also the higher flux in the FUV.

possible. In order to define the continuum in crowded regions, we measured equivalent widths of all unblended lines and then generated synthetic spectra using the abundances derived from this analysis; the continuum of the crowded regions was set by matching the synthetic spectra to the observations using a "chiby-eye" fit. This was not always straightforward however, since many lines still suffer from imprecise atomic data, and some resonance line profiles are not well reproduced in our assumed LTE atmosphere (see Sect. 5.1 for more discussion and examples of this effect). Nevertheless there were almost always enough useful lines for our method to be successful. Note that we are not relying on precise knowledge of the star's $U, B$, or $V$ magnitude - which is not always available; PG $1219+534$ is one case - to calibrate the model flux.

\subsection{Unblended lines}

There are various line lists available for the analysis of stellar spectra; most commonly used is the Kurucz list, although during our analysis we found that often more accurate oscillator strengths and wavelengths are available in other databases (e.g. Hirata \& Horaguchi 1995). In particular we found that several Co lines had poor term identifications in the Kurucz list, $\mathrm{Fe}$ oscillator strengths were often out of date, while oscillator strengths for Ti III were completely inconsistent with our observations.

To determine which lines in our spectra are not blended, we generated synthetic spectra using only those lines in the Kurucz list that have been observed experimentally and compared these directly with our observations. If the continuum was clear on either side of the line wings, and our line list showed only one possible match, we considered it to be unblended. Lines that were visibly blended (e.g. with overlapping wings) were ruled out for equivalent width measurement where the blend could not be identified.

\subsection{Individual elements}

In many regions of our spectra, line blending is extremely severe, which means that continuum definition is difficult (as discussed above) and/or that many features are identified with more than one ion. In this section we discuss the ions we have identified.

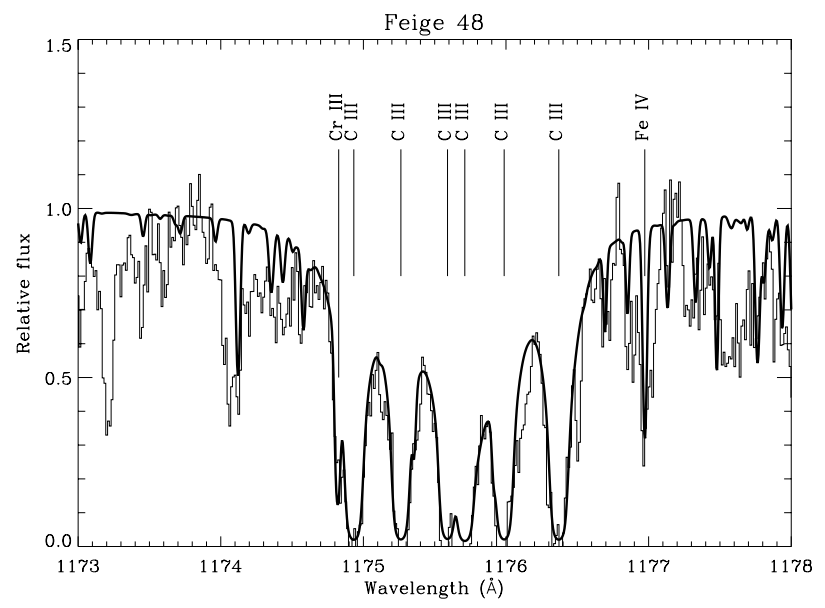

Fig. 4. Spectrum fit to the C III multiplet at 1174-1177 ̊ for Feige 48. The model is shown as the thick line. Other strong lines due to Cr IV and Fe IV are marked.

\subsection{1. $\mathrm{C}$ and $\mathrm{N}$}

PG $1219+534$ and CD $-24^{\circ} 731$ do not show any carbon lines. In CPD $-64^{\circ} 481$ the C III lines at 1247 and $2297 \AA$ are present, along with the $1176 \AA$ multiplet, while in Feige 48 and Feige 66 these C III lines and the C IV resonance lines around $1550 \AA$ are seen. Figure 4 shows the $1176 \AA$ multiplet of Feige 48 together with a model spectrum fit; results from spectrum fitting are discussed further in Sect. 5.

As found in almost all other sdBs, nitrogen lines are strong in our spectra, although there are fewer lines than seen in optical spectra. In our two cooler targets, N III lines are visible at $1183-1184 \AA$ while the N IV line at $1718 \AA$ is present in Feige 48. In Feige 66, PG 1219+534 and CD -24 731 , lines of $\mathrm{N}$ III and N IV, as well as the $\mathrm{N} V$ resonance lines can be seen.

\subsubsection{Al and $\mathrm{Si}$}

As has been noted by several authors (e.g. Lamontagne et al. $1985,1987)$, the abundance of silicon in sdB stars drops sharply at $\sim 32000 \mathrm{~K}$. This is also the case for aluminum, although the available upper limits on abundances are not as strict as for silicon.

Silicon in hot subdwarfs has been discussed in more detail recently by O'Toole (2004) and the objects studied here follow previously seen trends. The detection of the Si IV resonance line in PG 1219+534 is discussed further in Sect. 5.3. The Al III resonance lines are only detectable in Feige 48, although for $\mathrm{CPD}-64^{\circ} 481$ the relevant wavelength range is not covered by our spectra.

\subsubsection{S, Ar, Ca}

The higher resolution of our spectrum of CPD $-64^{\circ} 481$ allows us to clearly distinguish the S III resonance lines (shown in Fig. 5). Because of severe crowding and lower resolution, this is not possible in the case of Feige 48. The crowding is less severe for PG 1219+534, Feige 66 and CD -24 731 - although in the latter the lines are weak - so it is possible to make out the S III lines.

Ar III lines are difficult to measure as they are all quite weak, and often blended with lines due to iron-group elements. 


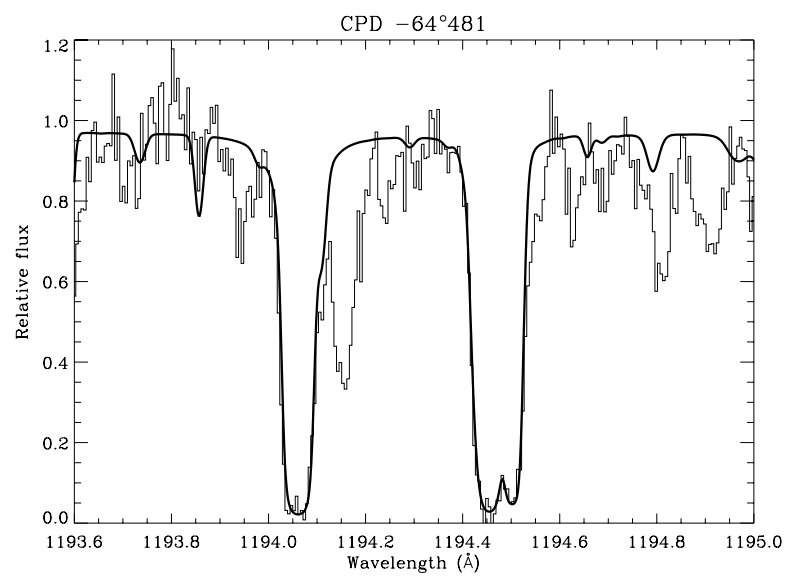

Fig. 5. The S III resonance lines for CPD $-64^{\circ} 481$, showing an excellent match. The line at $\sim 1194.5 \AA$ is one of the Si II resonance lines, while the strong line at $\sim 1194.15 \AA$ is unidentified.

Despite this, we could measure four lines in Feige 66 and one in PG 1219+534; the other stars have only upper limits.

In the case of calcium, we were able to measure lines of Ca III for Feige $66, \mathrm{CD}-24^{\circ} 731$ and PG $1219+534$. In the cooler pair the $\mathrm{Ca}$ lines are either very weak or blended, making measurements difficult.

\subsubsection{The iron group}

Lines of the iron group elements often lie at similar wavelengths, making it difficult to measure equivalent widths, and necessitating an analysis by spectrum synthesis. An example of this can be seen in the $4 \AA$-wide spectrum slices shown in Fig. 6 .

Scandium: there are very few Sc III lines available to measure in our spectra, making abundances very difficult to measure. It was only possible to measure the resonance lines at $1603.064 \AA$ and $1610.194 \AA$ for Feige 66 and CD $-24^{\circ} 731$. For Feige 48 and PG $1219+534$ we could only place upper limits, and our spectrum of CPD $-64^{\circ} 481$ does not cover these lines.

Titanium: the Ti III resonance lines are present in the spectrum of each star. Some subordinate lines are also measurable. We note here that the oscillator strengths found in the Kurucz line list are inconsistent with our observations. Therefore we have used the values given by Raassen \& Uylings (1997), which match significantly better.

Vanadium: lines of V III are typically blended or weak; no lines could be detected at all for Feige 48, allowing only upper limits to be set.

Chromium: all of our spectra contain many lines of both Cr III and CrIV of varying strengths. These lines, along with those of iron, have been used to determine the microturbulence discussed in Sect. 5.2.

Manganese: of all the iron-group elements, manganese causes the largest difficulties, since it appears that many wavelengths given in the Kurucz list are not accurate (note that the Hirata \& Horaguchi 1995, list uses the same wavelengths). We have examined the list of Uylings \& Raassen (1997), however they only provide improved oscillator strengths, and also use the Kurucz wavelengths.

Iron: in all spectra there are many Fe III and Fe IV lines measurable. In the two hottest objects in our sample, Fe V lines are also present, although the accuracy of oscillator strengths is

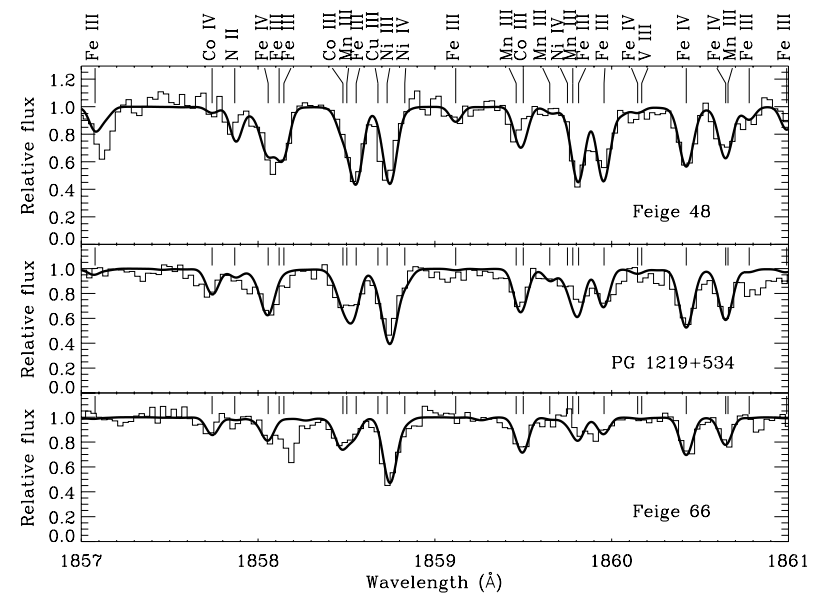

Fig. 6. A region of spectrum showing blended iron-group lines for Feige 48, PG $1219+534$ and Feige 66. The line at $\sim 1858.2 \AA$ is unidentified.

uncertain, since the abundances derived from some of them differ by several orders of magnitude.

Cobalt: lines of Co III and IV are visible in our spectra, although often only in crowded regions. It appears that the hotter objects show more strong lines.

Nickel: there are very many nickel lines, particularly of Ni III and IV. A few Ni V lines are also visible in the spectra of PG 1219+534 and Feige 66, however once again the accuracy of oscillator strengths is very uncertain.

Copper: Cu III and IV are both present. Neither ion is included in the Kurucz list, so wavelengths were taken from Hirata \& Horaguchi (1995). Oscillator strengths are only available for $\mathrm{Cu}$ III. There are no published oscillator strengths of $\mathrm{Cu}$ IV.

Zinc: Zn III and IV lines are visible; however, the latter ion has no published oscillator strengths.

\subsubsection{Gallium, germanium, tin and lead}

The discovery of lines of $\mathrm{Ga}, \mathrm{Ge}, \mathrm{Sn}$ and $\mathrm{Pb}$ was made by O'Toole (2004). Lines of Ge IV and Sn IV have also been seen in hot DA white dwarfs by Vennes et al. (2005). Abundances for our four targets are almost all done using spectrum synthesis. Resonance lines of Ga III, Ge IV, Sn IV, and Pb IV are present in all spectra. In stars with higher Ga abundances, Ga III subordinate lines are also present. The resonance line of Sn III at 1251.387 $\AA$ is strong in Feige 66, and is present in all stars, but is blended.

One of the resonance lines of $\mathrm{Pb}$ IV is present in each object; the other line lies in the wings of $\operatorname{Ly} \beta$, which is not covered by our spectra (but is by FUSE).

\section{Abundance analysis}

In this section we comment on results and trends for individual or groups of elements. The abundances are given in Table 2 and plotted relative to the sun in Fig. 9. The errors given were determined using a simple mean and standard deviation based on the individual measurement for each line. An error of zero represents a measurement taken from only one line. The solar values were taken from Grevesse \& Sauval (1998). The O, $\mathrm{Ne}$ and $\mathrm{Mg}$ abundances of the programme stars are taken from HRW for PG $1219+534$ and Feige 48 , while for CD $-24^{\circ} 731$ 
Table 2. Abundances for each ion for all five targets.

\begin{tabular}{|c|c|c|c|c|c|c|c|c|c|c|}
\hline Ion & $\begin{array}{c}\text { Feige } 66 \\
\log \epsilon\end{array}$ & $n$ & $\begin{array}{c}\text { PG 1219+534 } \\
\log \epsilon \\
\end{array}$ & $n$ & $\begin{array}{c}\mathrm{CD}-24^{\circ} 731 \\
\log \epsilon\end{array}$ & $n$ & $\begin{array}{c}\text { Feige } 48 \\
\log \epsilon\end{array}$ & $n$ & $\begin{array}{c}\mathrm{CPD}-64^{\circ} 481 \\
\log \epsilon \\
\end{array}$ & $n$ \\
\hline C III & $6.93 \pm 0.28^{*}$ & 4 & & & & & $7.22 \pm 0.10$ & 3 & $7.54 \pm 0.22$ & 2 \\
\hline C IV* & $6.5 \pm 0.5$ & 2 & $<3.1$ & & $<2.7$ & & $6.9 \pm 0.5$ & & & \\
\hline N II & & & & & & & & & $7.87 \pm 0.14$ & 3 \\
\hline N III & $7.69 \pm 0.23$ & 2 & $7.64 \pm 0.42$ & 4 & $7.60 \pm 0.47$ & 6 & $7.62 \pm 0.38$ & 3 & $7.40 \pm 0.00$ & 2 \\
\hline NIV & $7.65 \pm 0.00$ & 1 & & & & & & & & \\
\hline $\mathrm{NV}$ & $7.60 \pm 0.11$ & 2 & $7.58 \pm 0.21$ & 2 & $8.26 \pm 0.15$ & 2 & & & & \\
\hline Al III & $<3.5$ & & $<3.8$ & & $<3.6$ & & $5.51 \pm 0.10$ & 3 & & \\
\hline Si III & & & & & & & $6.43 \pm 0.31$ & 8 & $6.38 \pm 0.37$ & 3 \\
\hline Si IV* & $<2.0$ & & $3.63 \pm 0.17$ & 2 & $<2.0$ & & $6.16 \pm 0.25$ & 3 & & \\
\hline P III & & & & & & & $4.41 \pm 0.00$ & 1 & $4.81 \pm 0.09$ & 3 \\
\hline PIV & $3.95 \pm 0.00$ & 1 & & & & & & & & \\
\hline S III & $7.69 \pm 0.46$ & 4 & $7.00 \pm 0.11$ & 3 & $5.7 \pm 0.00$ & 1 & & & $6.29 \pm 0.27$ & 3 \\
\hline Ar III & $7.86 \pm 0.24$ & 4 & $7.02 \pm 0.00$ & 1 & $<7.0$ & & $<7.0$ & & & \\
\hline Ca III & $8.09 \pm 0.20$ & 20 & $7.73 \pm 0.23$ & 10 & $7.50 \pm 0.22$ & 8 & $7.31 \pm 0.00$ & 1 & $6.97 \pm 0.00$ & 1 \\
\hline Sc III & $5.17 \pm 0.51$ & 2 & $<5.0$ & & $4.61 \pm 0.00$ & 1 & $<3.0$ & & & \\
\hline Ti III & $6.98 \pm 0.22$ & 10 & $6.51 \pm 0.20$ & 2 & $5.77 \pm 0.06$ & 3 & $5.16 \pm 0.20$ & 4 & $5.68 \pm 0.37$ & 6 \\
\hline Ti IV & $6.91 \pm 0.18$ & 5 & $6.73 \pm 0.00$ & 1 & $5.87 \pm 0.15$ & 3 & $5.21 \pm 0.18$ & 2 & $5.75 \pm 0.07$ & 2 \\
\hline V III & $6.42 \pm 0.22$ & 11 & $5.80 \pm 0.00$ & 1 & & & & & $4.48 \pm 0.15$ & 4 \\
\hline V IV & $6.29 \pm 0.14$ & 7 & $6.37 \pm 0.34$ & 2 & $5.77 \pm 0.37$ & 9 & & & & \\
\hline Cr III & $7.29 \pm 0.19$ & 65 & $7.23 \pm 0.17$ & 44 & $6.96 \pm 0.23$ & 44 & $5.90 \pm 0.18$ & 29 & $6.20 \pm 0.30$ & 33 \\
\hline CrIV & $7.31 \pm 0.23$ & 25 & $7.46 \pm 0.14$ & 17 & $7.24 \pm 0.47$ & 28 & $6.41 \pm 0.28$ & 7 & $6.54 \pm 0.20$ & 10 \\
\hline Mn III & $6.02 \pm 0.19$ & 12 & $7.25 \pm 0.22$ & 46 & $6.89 \pm 0.36$ & 19 & $5.62 \pm 0.21$ & 13 & $5.54 \pm 0.21$ & 15 \\
\hline Mn IV & $5.99 \pm 0.51$ & 2 & $7.72 \pm 0.11$ & 3 & $7.12 \pm 0.54$ & 7 & & & $5.86 \pm 0.18$ & 2 \\
\hline $\mathrm{Fe}$ III & $6.46 \pm 0.17$ & 26 & $7.16 \pm 0.22$ & 69 & $6.66 \pm 0.26$ & 23 & $7.68 \pm 0.19$ & 107 & $7.38 \pm 0.33$ & 20 \\
\hline Fe IV & $6.40 \pm 0.10$ & 2 & $7.43 \pm 0.22$ & 23 & $7.03 \pm 0.25$ & 23 & $7.73 \pm 0.21$ & 10 & $7.52 \pm 0.23$ & 11 \\
\hline $\mathrm{Fe} \mathrm{V}$ & & & $7.38 \pm 0.41$ & 2 & & & & & & \\
\hline Co III & $6.46 \pm 0.33$ & 39 & $6.73 \pm 0.20$ & 20 & $6.13 \pm 0.39$ & 20 & $5.73 \pm 0.25$ & 5 & & \\
\hline Co IV & $6.32 \pm 0.22$ & 20 & $6.72 \pm 0.13$ & 7 & $6.28 \pm 0.21$ & 11 & $6.05 \pm 0.25$ & 3 & & \\
\hline Ni III & $6.86 \pm 0.15$ & 32 & $7.39 \pm 0.18$ & 32 & $6.20 \pm 0.48$ & 12 & $6.63 \pm 0.22$ & 28 & $6.39 \pm 0.25$ & 6 \\
\hline Ni IV & $6.81 \pm 0.25$ & 32 & $7.41 \pm 0.27$ & 19 & $6.28 \pm 0.28$ & 14 & $6.75 \pm 0.21$ & 7 & $6.50 \pm 0.26$ & 9 \\
\hline $\mathrm{Niv}$ & $6.91 \pm 0.55$ & 10 & $7.60 \pm 0.14$ & 5 & & & & & & \\
\hline $\mathrm{Cu}$ III & $6.27 \pm 0.38$ & 8 & $6.36 \pm 0.36$ & 5 & & & $5.25 \pm 0.40$ & 3 & & \\
\hline Zn III & $6.64 \pm 0.35$ & 15 & $6.68 \pm 0.36$ & 5 & $5.97 \pm 0.50$ & 2 & $5.30 \pm 0.22$ & 6 & $5.33 \pm 0.34$ & 6 \\
\hline Ga III & $5.53 \pm 0.06$ & 2 & $5.75 \pm 0.04$ & 2 & $5.23 \pm 0.21$ & 2 & $4.70 \pm 0.00$ & 1 & $3.63 \pm 0.00$ & 1 \\
\hline Ge IV & $5.21 \pm 0.05$ & 2 & $4.99 \pm 0.03$ & 2 & $4.98 \pm 0.01$ & 2 & $4.02 \pm 0.08$ & 2 & $3.57 \pm 0.10$ & 2 \\
\hline Sn IV & $4.12 \pm 0.00$ & 1 & $4.08 \pm 0.00$ & 1 & $3.11 \pm 0.00$ & 1 & $2.94 \pm 0.00$ & 1 & $2.86 \pm 0.00$ & 1 \\
\hline $\mathrm{Pb} I V$ & $4.7 \pm 0.00$ & 1 & $4.6 \pm 0.00$ & 1 & $4.3 \pm 0.00$ & 1 & $3.8 \pm 0.00$ & 1 & $3.43 \pm 0.00$ & 1 \\
\hline
\end{tabular}

${ }^{*}$ Based on non-LTE-affected resonance lines; see Sect. 5.1.

and CPD $-64^{\circ} 481$, they are from Edelmann (private communication), along with the $\mathrm{Al}$ abundance for the latter star.

Before discussing our derived abundances in more detail, however, it is necessary to first consider non-LTE effects and the effect of microturbulence on the absorption lines in our spectra.

\subsection{NLTE effects}

Most of the spectral lines we are dealing with in this paper are subordinate, i.e. they are between excited levels and do not involve the ground level. The resonance lines (transitions from the ground level) of some ions are present however, and it is these lines that are most sensitive to departures from our assumption of local thermodynamic equilibrium (LTE). Ions of iron-group elements do not appear to be affected, but species such as C IV and Si IV, there are noticeable effects. In Fig. 8 we show the C IV resonance lines at $1548 \AA$ and $1550 \AA$ of Feige 66 as an example. In this star and Feige 48 the line cores of these lines are matched by the abundances derived from the $\mathrm{C}$ III lines, however the wings are much too broad. Reducing the $\mathrm{C}$ abundance results in an improved fit in the wings but a core that is too shallow. This is most likely a non-LTE effect. Detailed model atom calculations are needed to confirm this, but are beyond the scope of this paper. There may also be an interstellar component present, at least in Feige 66, which has a radial velocity of only $\sim-6 \mathrm{~km} \mathrm{~s}^{-1}$; however, this is not the case for Feige 48, and the effect is still present.

In the case of $\mathrm{C}$ III all three lines/multiplets can be matched well with a model spectrum at the listed abundance for Feige 48, however for Feige 66 things are not so simple. The 1174-1177 multiplet is apparently matched well; the $1247 \AA$ line is not, while the $2297 \AA$ line matches in the wings but not in the line core. Because Feige 66 is around $5000 \mathrm{~K}$ hotter than Feige 48, we again attribute these problems to non-LTE effects.

The iron-group lines we have used for our abundance analysis are not resonance lines (with the exception of Ti III where some resonance lines were used), so are not likely to be strongly affected by non-LTE effects.

\subsection{Microturbulence}

Since we have measured the equivalent widths of many lines for several ions, we can investigate the microturbulence $\xi$ for each star. Both of the pulsating sdBs, along with CPD $-64^{\circ} 481$, 

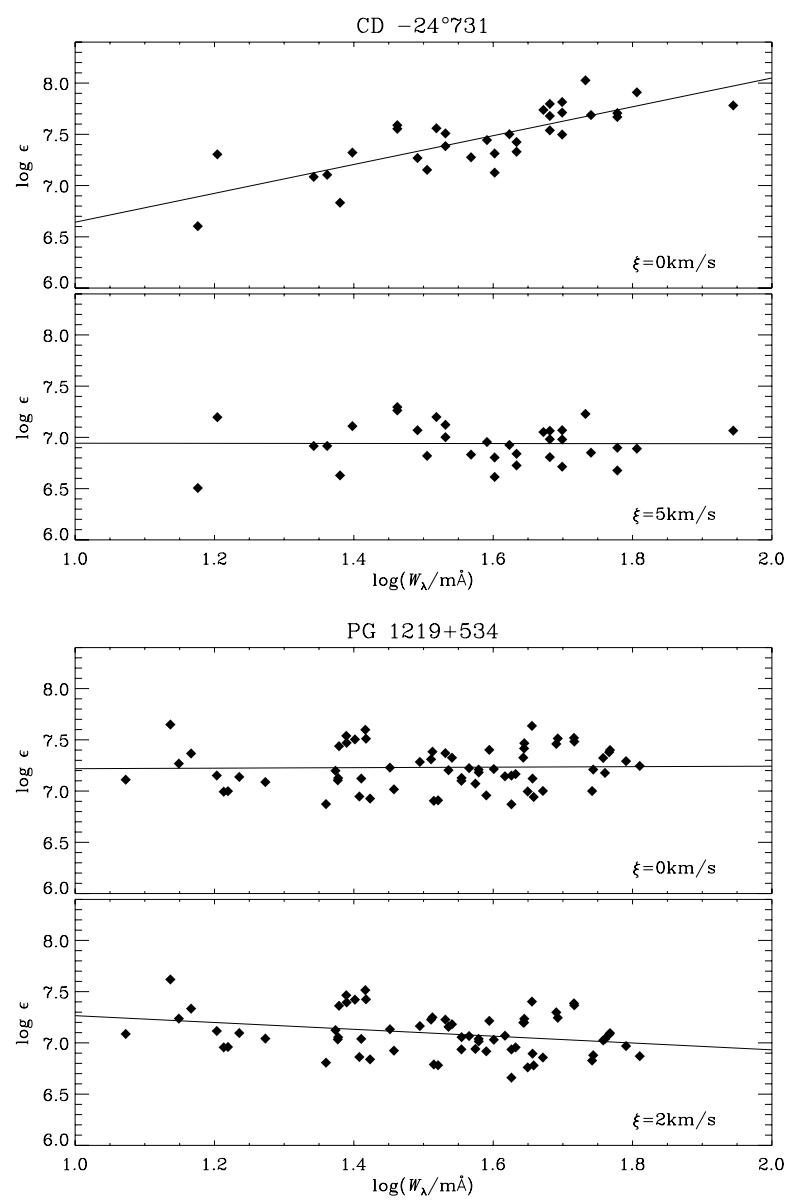

Fig. 7. Determination of microturbulent velocity of $C D-24^{\circ} 731$ and PG $1219+534$ using Cr III lines. We find $\xi=5_{-1}^{+2} \mathrm{~km} \mathrm{~s}^{-1}$ for the former, while the latter is consistent with $\xi=0 \mathrm{~km} \mathrm{~s}^{-1}$.

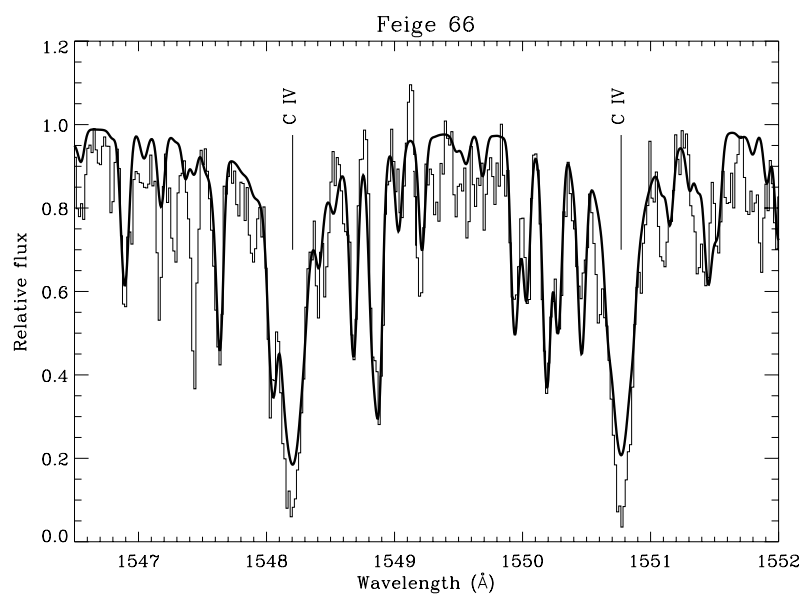

Fig. 8. Spectrum fit to the C IV resonance lines at $1550 \AA$ for Feige 66 . The model is shown as the thick line. The line cores of the model do not match the observations, and the abundance is set around 0.8 dex lower than that derived from C III lines. See text for details.

are consistent with $\xi=0 \mathrm{~km} \mathrm{~s}^{-1}$; however, the two other nonpulsators both have $\xi>0 \mathrm{~km} \mathrm{~s}^{-1}$. The top panels of Fig. 7 shows the difference between $\xi=0 \mathrm{~km} \mathrm{~s}^{-1}$ and the value we derive $\xi=$ $5_{-1}^{+2} \mathrm{~km} \mathrm{~s}^{-1}$ for CD $-24^{\circ} 731$; the bottom panels show the effect of non-zero microturbulence for PG 1219+534. For Feige 66 we find $\xi=2 \pm 1 \mathrm{~km} \mathrm{~s}^{-1}$. These values have been derived using both Cr III and Fe III lines.

\subsection{Abundances}

Before looking at the differences in iron-group abundances of our targets, we first search for and examine trends amongst the lighter elements.

\subsubsection{Light metals}

As has been found by many previous studies, carbon abundances range from virtually none at all to slightly below the solar value. For PG $1219+534$ and CD $-24^{\circ} 731$ the absence of the C IV resonance lines indicates carbon depletion by $10^{5}$ or more. Because we cannot at the moment account for NLTE effects (see Sect. 5.1 above) these have to be regarded as order of magnitude estimates. We note here that simple NLTE calculations were done by Heber et al. (1984a) for the sdO star Feige $110\left(T_{\text {eff }}=40000 \mathrm{~K}\right)$ and a similar upper limit was derived.

In the case of nitrogen, the abundances are slightly below the solar value; this is also in keeping with previous analyses of sdB optical spectra. We note in passing that we could not easily fit the N IV line at $1718.551 \AA$ in all of our sample because of severe crowding - only in Feige 66 is the line isolated - however the strength of the line is approximately consistent with the abundances derived from the other $\mathrm{N}$ ionisation stages. Note that the nitrogen abundances from three different ionisation stages are fully consistent for Feige 66.

For Feige 48 it was not possible to measure the sulfur abundance because the resonance lines are blended with a large number of metal lines that are not in the Kurucz list of observed lines or the Hirata \& Horaguchi list. This effect is seen to a lesser extent in PG 1219+534, making a spectrum fit possible, and hardly seen at all in Feige 66. Because of the absence of heavy crowding in the latter star, we suggest these may be iron lines. We examined the effect of including the "computed" (but not observed) lines in the Kurucz list: the match is better for Feige 48, but it is still not possible to measure abundances using the S III lines.

\subsubsection{Iron group}

As can be seen from Table 2, plenty of lines of doubly ionised atoms (in particular Cr III, Fe III and Ni III) have been used for the abundances analysis, for Feige 48 more than $100 \mathrm{Fe}$ III lines were utilised. Moreover, triply ionised atoms can be used to determine abundances for all iron group elements as well (except for V IV and Mn IV in Feige 48 and V IV, and Co IV in CPD -64 ${ }^{\circ} 481$ ), which in some stars are also quite numerous (e.g. $32 \mathrm{Ni} \mathrm{IV} \mathrm{lines}$ in Feige 66). Even four times ionised atoms have been used for analysis of nickel in Feige 66 and PG 1219+534 and of iron in PG $1219+534$. It is worthwhile noting that these ionisation equilibria are very well matched, e.g. the three ionisation stages of Ni agree to within 0.1 dex for Feige 66 and to within 0.2 dex for PG 1219+534. For other ions of the iron group, we find:

- Fe III and Fe IV as well as Ni III and Ni IV, respectively, agree very well ( 0.1 dex, typically) for Feige 48 and CPD $-64^{\circ} 481$ and to within error limits in $\mathrm{CD}-24^{\circ} 731$ ( 0.37 dex for iron).

- CrIII and CrIV agree to better than 0.3 dex except for Feige 48 ( 0.5 dex $)$.

- Mn III and Mn IV are in perfect agreement for Feige 66, differ by about 0.3 dex in $C D-24^{\circ} 731$ and CPD $-64^{\circ} 481$. Only for PG 1219+534 they deviate beyond the adopted error ranges.

- Co III and Co IV also match very well, to better than 0.2 dex, except for Feige 48 which has much less Co lines than the other programme stars. 


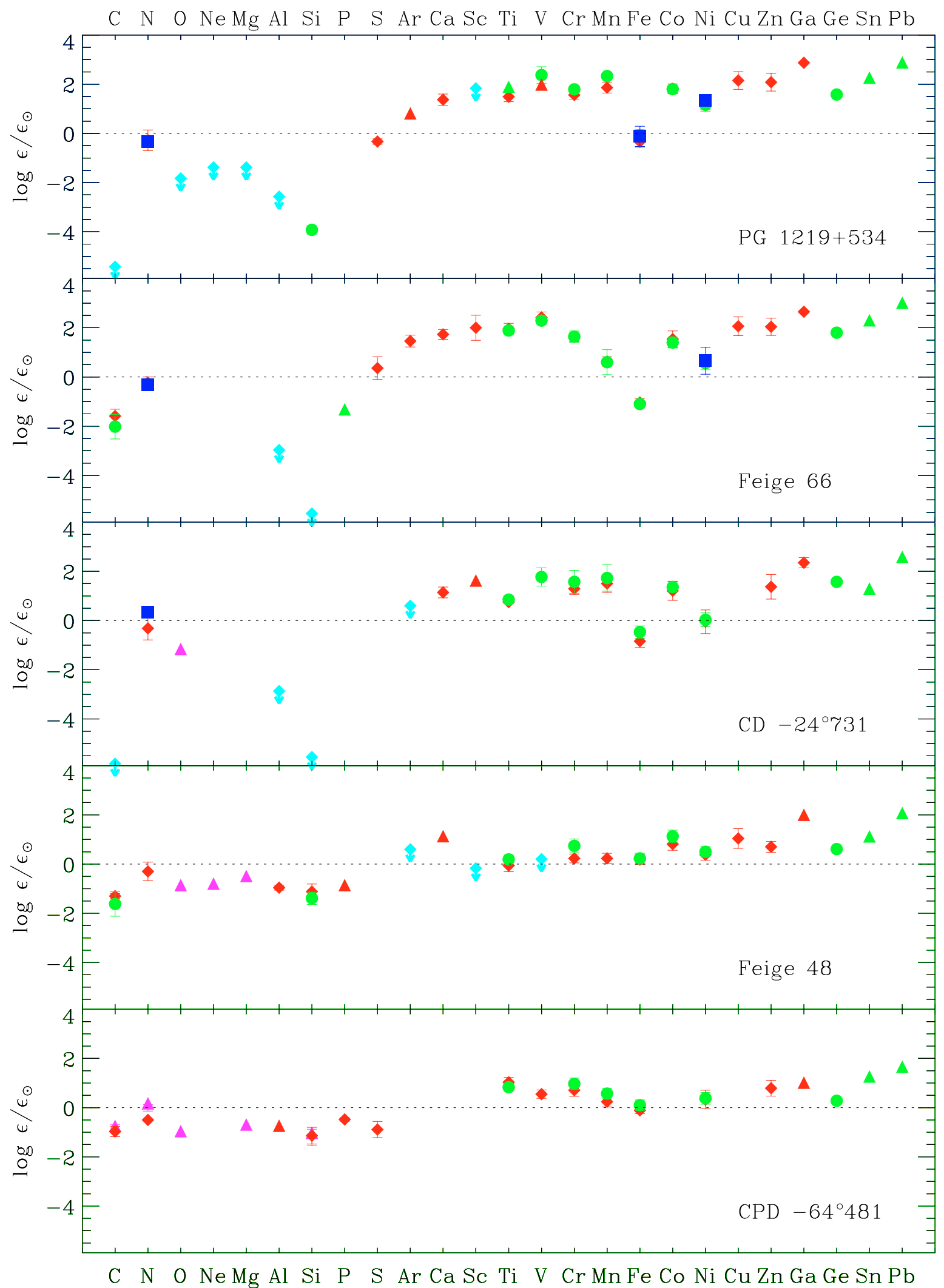

Fig. 9. Abundances measured for our five targets. Magenta symbols represent values determined using singly ionised lines, green represents doubly ionised lines, red represents triply ionised lines, blue denotes quadruply ionised lines and cyan represents upper limits. Note the generally excellent agreement between different ionisation stages.

- Although only few titanium lines could be used for the analysis, the results from Ti III and Ti IV match extremely well for all programme stars.
We regard the very good match of many ionisation equilibria as evidence that systematic errors of the metal abundances and of the effective temperature are small. This can also be seen in 


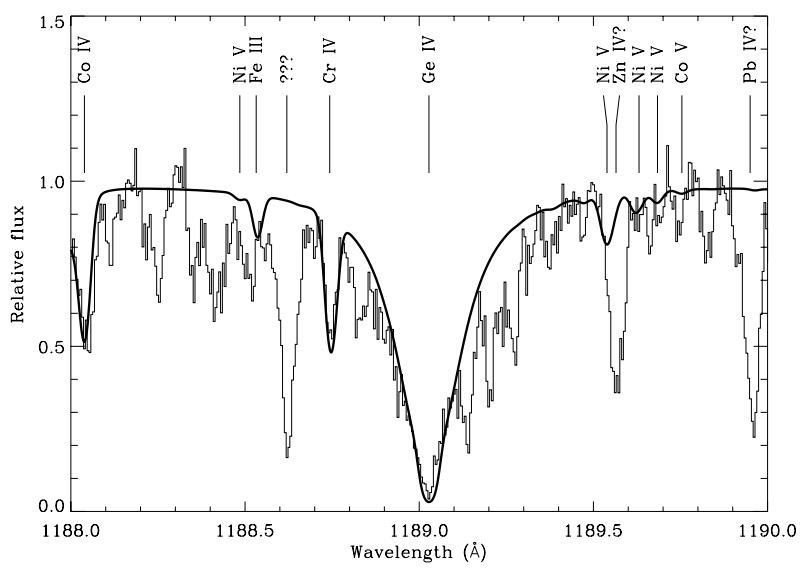

Fig. 10. Spectrum fit to the Ge IV resonance lines at $1189 \AA$ A for Feige 66 . The model is shown as the thick line.

the good match between synthetic spectrum and observations for three objects shown in Fig. 6.

While iron is found to be nearly solar (PG 1219+534, Feige 48, CPD $-64^{\circ} 481$ ), slightly depleted in CD $-24^{\circ} 731$ and subsolar in Feige 66 by a factor of ten, all other elements of the iron group are enhanced by between 0.5 and 2.5 dex with respect to solar values. The enhancements are large in Feige 66 and PG $1219+534$, but mild for the three others.

\subsubsection{Gallium, germanium, tin and lead}

The heavy metals $\mathrm{Ga}, \mathrm{Ge}, \mathrm{Sn}$ and $\mathrm{Pb}$ are all enriched with respect to the Sun in all stars, reaching as high as 2.9 dex for $\mathrm{Ga}$ in PG $1219+534$ or 2.75 dex for $\mathrm{Pb}$ in Feige 66. An example of a fit of the Ge IV $1189 \AA$ line is shown in Fig. 10. There are several strong lines also present that are either not identified or have no atomic data. Possible identifications are shown in the figure.

\subsection{Comparison with previous work}

As mentioned earlier, many of the objects we have observed for this project have been studied with optical spectra before - PG 1219+534 and Feige 48 (Heber et al. 2000), and CPD $-64^{\circ} 481$ and $\mathrm{CD}-24^{\circ} 731$ (Edelmann, private communication) - or with a noisy IUE spectrum and poor atomic data Feige 66 (Baschek et al. 1982a). It is useful here to compare our results with these previous studies, as well as with any general trends seen amongst sdBs as a group.

Firstly, for the two pulsators PG $1219+534$ and Feige 48, our results compare very well with those of HRW for the limited cross-over that exists between elements. All of HRW's abundances are, within errors, consistent with ours, and because we could use resonance lines of several ions, we have been able to place stronger upper limits on C, Al and Si for PG 1219+534. In the case of Feige 48 the abundances we derive agree well with those measured by HRW, and are within \pm 0.04 dex.

The only element we have had difficulty deriving an abundance for is sulfur, for the reasons discussed earlier.

In the case of Feige 66, Baschek et al. (1982a) used IUE spectra with lower resolution than ours, and obtained somewhat different results to ours. It is difficult to compare the two sets of abundances since Baschek et al. do not give error estimates explicitly; however, if we assume the same errors as those given for another sdB, HD 149382, in the same paper, our values are not so discrepant after all. At the time of Baschek et al. study, the

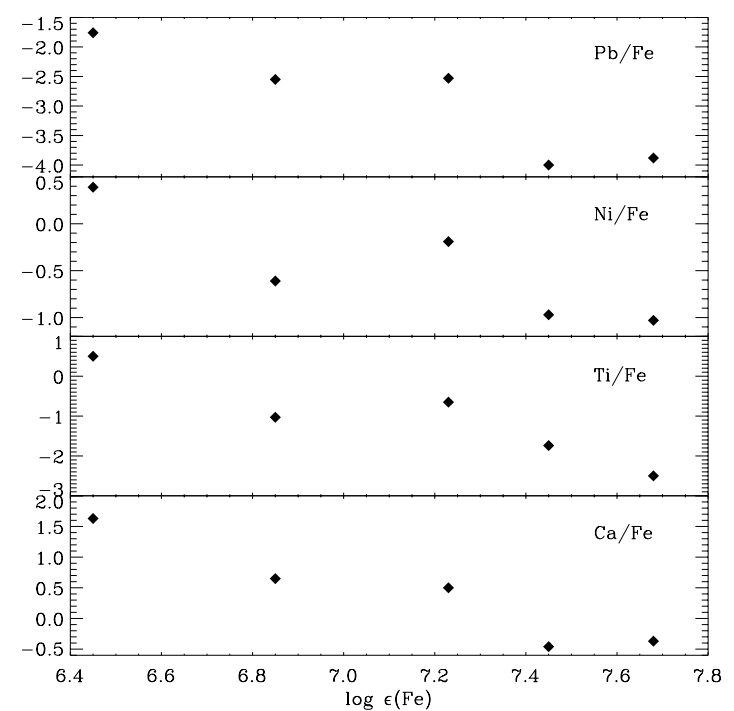

Fig. 11. Trends in heavy element abundances with iron abundance.

quality of atomic data was lower than it is at present (although in many cases it is still insufficient or of low accuracy), so their errors are very large, particularly for the iron group.

Recently, Edelmann et al. (2006) has carried out an analysis of CPD $-64^{\circ} 481$ and CD $-24^{\circ} 731$ based on high-resolution optical echelle spectra. For both stars their abundances are consistent with those determined here.

Finally, we can also compare any apparent trends seen here with the work of Heber \& Edelmann (2004), especially for the lighter elements plus iron. Both our results here and the work of Heber \& Edelmann (2004) suggests that aluminum follows the same trend as silicon.

\subsection{Heavy element abundance trends?}

An interesting result not immediately obvious from the abundances in Table 2 is a possible anti-correlation between heavy element abundance and iron abundance. The spread in iron abundance is 1.25 dex from the most iron-poor (Feige 66) to the most iron-rich star (Feige 48), while the variation (X/Fe) is between 2 and 3 dex (except for $\mathrm{Mn}$ and $\mathrm{Ni}$ ). Therefore this trend does not simply reflect the trend in iron abundance. Four examples of this effect are shown in Fig. 11 for $\mathrm{Ca} / \mathrm{Fe}, \mathrm{Ti} / \mathrm{Fe}, \mathrm{Ni} / \mathrm{Fe}$ and $\mathrm{Pb} / \mathrm{Fe}$ while Table 3 shows data for all elements; where there are two or more ionisation stages, a weighted average is presented. The same basic trend is seen for all elements in the iron-group, except for manganese and perhaps nickel, and it is also seen for the heavier elements gallium, germanium, tin and lead. Caution must be applied here however, since we have only five objects in our sample. A more detailed analysis may be possible for at least titanium, since several sdB stars show Ti III lines in their optical spectra (Edelmann, Karl, private communication). If these trends are real we believe they are caused by a combination of radiative levitation, gravitational settling and a weak stellar wind, and therefore would help constrain diffusion models. We urge more theoretical work in this area.

\subsection{Comparison of abundances of pulsator/non-pulsator pairs}

The results of our spectral analysis allow us to compare the abundance patterns of four pairs of stars, having similar 
Table 3. Average iron abundance and ratio of heavy element abundances to iron for the five targets stars. The columns are as follows: $\mathrm{F} 66=$ Feige 66; $\mathrm{CD}=\mathrm{CD}-24^{\circ} 731 ; \mathrm{PG}=\mathrm{PG} 1219+534$; $\mathrm{CPD}=\mathrm{CPD}-64^{\circ} 481 ; \mathrm{F} 48=$ Feige 48 . Abbreviations: $\mathrm{n}-\mathrm{v}=$ nonvariable, var. $=$ variable

\begin{tabular}{l|rrr|rr}
\hline \hline & F66 & CD & PG & CPD & F48 \\
& n-v & n-v & var. & n-v & var. \\
$\mathrm{Fe}$ & 6.46 & 6.85 & 7.23 & 7.43 & 7.68 \\
\hline $\mathrm{Ca} / \mathrm{Fe}$ & 1.63 & 0.65 & 0.50 & -0.46 & -0.37 \\
$\mathrm{Ti} / \mathrm{Fe}$ & 0.50 & -1.03 & -0.65 & -1.74 & -2.50 \\
$\mathrm{~V} / \mathrm{Fe}$ & -0.09 & -1.08 & -1.08 & -2.95 & - \\
$\mathrm{Cr} / \mathrm{Fe}$ & 0.84 & 0.23 & 0.06 & -1.15 & -1.68 \\
$\mathrm{Mn} / \mathrm{Fe}$ & -0.44 & 0.10 & 0.05 & -1.85 & -2.06 \\
$\mathrm{Co} / \mathrm{Fe}$ & -0.05 & -0.69 & -0.50 & - & -1.83 \\
$\mathrm{Ni} / \mathrm{Fe}$ & 0.39 & -0.61 & -0.19 & -0.97 & -1.03 \\
$\mathrm{Cu} / \mathrm{Fe}$ & -0.19 & - & -0.87 & - & -2.43 \\
$\mathrm{Zn} / \mathrm{Fe}$ & 0.18 & -0.88 & -0.59 & -2.10 & -2.38 \\
$\mathrm{Ga} / \mathrm{Fe}$ & -0.93 & -1.62 & -1.70 & -3.80 & -2.98 \\
$\mathrm{Ge} / \mathrm{Fe}$ & -1.25 & -1.87 & -2.02 & -3.86 & -3.66 \\
$\mathrm{Sn} / \mathrm{Fe}$ & -2.34 & -3.74 & -3.11 & -4.57 & -4.74 \\
$\mathrm{~Pb} / \mathrm{Fe}$ & -1.76 & -2.55 & -2.53 & -4.00 & -3.88 \\
\hline
\end{tabular}

effective temperature: (i) the "cool" pulsator/non-pulsator pair Feige 48/CPD $-64^{\circ} 481$; (ii) the "hot" pulsator/non-pulsator pair PG 1219+534/CD -24 731; (iii) the "hot" pulsator/non-pulsator pair PG 1219+534/Feige 66 and (iv) the pair of two "hot" nonpulsators Feige $66 / \mathrm{CD}-24^{\circ} 731$. We plot the relative iron group abundances for all four combinations in Fig. 12).

(i) If we compare Feige 48 and CPD $-64^{\circ} 481$ (the bottom panel of Fig. 12), we find basically no difference. Not only are the abundances of the iron group elements similar, but the lighter and heavier elements also agree reasonably well. The only exception is gallium which is ten times higher in Feige 48 than in CPD $-64^{\circ} 481$. Also looking at abundance ratios of iron group elements with respect to iron $(\mathrm{X} / \mathrm{Fe}$, Table 3$)$ reveals, that the patterns for Feige 48 and CPD $-64^{\circ} 481$ match each other reasonably well. This would indicate that there is no difference in iron group abundances of a pulsator and a non-pulsator.

(ii) The comparison of "hot" pulsator PG 1219+534 to the nonpulsator CD $-24^{\circ} 731$ reveals large differences for some light elements, silicon and sulphur being much less in CD $-24^{\circ} 731$ (more than 1.6 dex and 1.3 dex, respectively). Significant differences are also detected for the iron group as well as for the heavy elements. They, however, vanish almost if the difference in iron content is accounted for (except for $\mathrm{Ni} / \mathrm{Fe}$ and $\mathrm{Sn} / \mathrm{Fe}$, see Table 3 ). Since the iron group elements are probably the drivers for pulsators, the comparison would indicate as in the previous case that there is little difference (if at all) between a pulsator and a non-pulsator.

(iii) The comparison of the "hot" pulsator PG 1219+534 to the non-pulsator Feige 66 yields a completely different picture. Abundances of several iron group elements differ (see Table 2). Even if we take into account the large difference in iron abundance (factor of 7) between the two stars, the abundance patterns remain dissimilar (see Table 3). Amongst the light elements there is a huge difference in carbon abundance. PG 1219+534 has less than 5000 times as much $\mathrm{C}$ as Feige 66 has. The abundances of the heavy elements ( $\mathrm{Ga}, \mathrm{Ge}, \mathrm{Sn}$, and $\mathrm{Pb}$ ), however, are quite similar. In contrast to the previous comparisons in (i) and (ii) this would imply that indeed pulsators and non-pulsators have different iron group abundances.

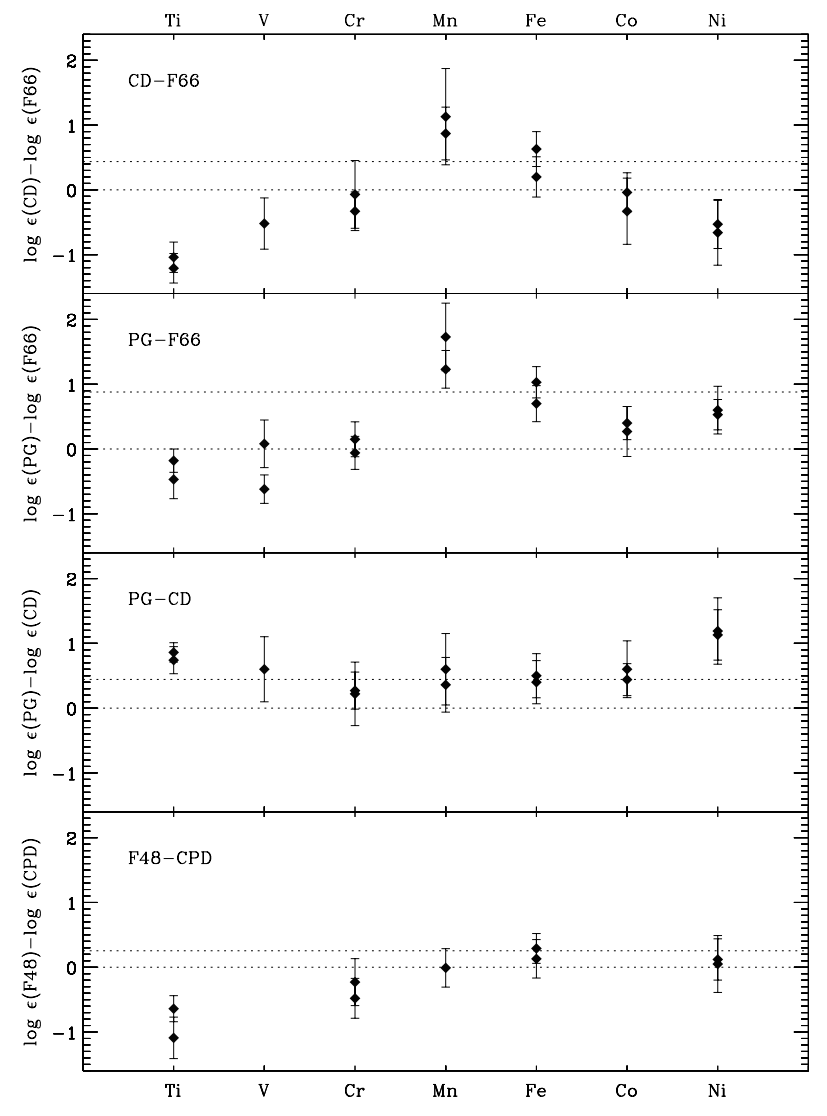

Fig. 12. Comparison of iron-group abundances for our pulsator/non-pulsator pairs (F48 = Feige 48; CPD $=\mathrm{CPD}-64^{\circ} 481$; $\mathrm{PG}=\mathrm{PG} 1219+534 ; \mathrm{CD}=\mathrm{CD}-24^{\circ} 731 ; \mathrm{F} 66=$ Feige 66$)$. The dotted lines denote equal abundances and the difference in iron abundance. The differences between PG $1219+534, \mathrm{CD}-24^{\circ} 731$ and Feige 66 are quite large - particularly for $\mathrm{Fe}, \mathrm{Ni}$ and $\mathrm{Mn}$ - while the pairs PG $1219+534$ and CD $-24^{\circ} 731$ and Feige 48 and CPD $-64^{\circ} 481$ show quite similar abundances, in particular when the differences in iron abundance are accounted for (see text).

(iv) The comparison of the two "hot" non-pulsators Feige 66 and $C D-24^{\circ} 731$ also reveals large differences in the abundances of iron group elements which persist if we account for the different iron abundance (see Table 3). Amongst the light elements as well as among the heavy elements there are large differences as well, which persist if we account for the different iron abundances.

The last finding is discouraging. If the difference among nonvariable stars of similar $T_{\text {eff }}$ and $\log g$ are as large as we observe, the comparison between a pulsator and a non-pulsator is rendered arbitrary.

The theory of Charpinet et al. (1997) does not specifically rule out iron-group elements other than iron itself as responsible for pulsation driving. In particular $\mathrm{Ni}$ must be considered as it has substantially more UV and FUV lines than iron. Indeed, in the case of PG 1219+534 the nickel abundance is high enough that it could make a significant contribution to the opacity, at least as much as iron. When we consider Feige 48 and $\mathrm{CPD}-64^{\circ} 481$, we find that perhaps the difference in the combined opacity of iron and nickel between the two stars is significant enough to discriminate between pulsator and non-pulsator. This leads us to ask: could we theoretically have a pulsator with lower iron, but much higher nickel or other iron-group abundance than a non-pulsator? 


\section{Further discussion}

As seen in Sect. 3.2, enhanced metal abundances can have an effect on $T_{\text {eff }}$ and $\log g$ determination, especially for stars showing both neutral and singly ionised helium. It is also clear that simply scaling models from solar metalicity ODFs is insufficient; opacity sampling is required for more accurate measurements, since while Fe abundances are approximately solar, elements such as $\mathrm{Ni}$ and $\mathrm{Mn}$ - which have a significant opacity contribution - are almost always enhanced. A preliminary study has been done in this direction by Behara \& Jeffery (2006) and Przybilla et al. (2006).

The effect of these abundance patterns may be apparent for atmosphere models, but what about stellar evolution and pulsation models? We urge evolution theorists to investigate the effect of non-solar opacity distributions on hot subdwarf evolution. In a similar vein, interior models that include differential internal rotation and/or magnetic fields should also investigate their effect on diffusion.

In a study of elements beyond the iron group in hot subdwarfs, O'Toole (2004) proposed a solution to the silicon problem discussed in Sect. 4.3.2. Triply ionised elements of the same group in the Periodic Table as silicon - germanium, tin and lead - are present in almost all sdB spectra at all temperatures. Si IV on the other hand, almost completely disappears above $32000 \mathrm{~K}$. This shows that arguments where silicon is ionised to nobe gas configuration, i.e. to $\mathrm{Si} \mathrm{V}$, and then sinks deeper into the atmosphere, cannot be correct. If this were the case, these heavier elements, which should feel the same low radiative forces as silicon, should also sink. Instead, O'Toole (2004) suggested that above $\sim 32000 \mathrm{~K}$ silicon could be carried away by a weak fractionated stellar wind, whereas the heavier elements should stay behind. Indeed, Unglaub (2006) has found that a one-component, uniform wind is inconsistent with observations, and that multicomponent calculations are required, although this depends on surface gravity and mass-loss rate. When we examine the abundances of $\mathrm{Ge}, \mathrm{Sn}$ and $\mathrm{Pb}$, we find that they are all higher in the hot stars (Feige 66, CD $-24^{\circ} 731$, and PG 1219+534) that show little or no silicon, than in the cooler stars (Feige 48, CPD $-64^{\circ} 481$ ) that do. This is what one would qualitatively expect from the hypothesis of O'Toole (2004). Using FUSE spectra, Chayer et al. (2006) found many other sdBs with similar properties, but also some exceptions. The case of C IV is not at all straightforward, since it is very difficult to explain two spectroscopically similar stars, one with measureable silicon but no carbon (PG 1219+534), and the other with measureable carbon but no silicon (Feige 66 ). CD $-24^{\circ} 731$ has no carbon and no silicon, compounding the problem. The reason for this remains a mystery, and presents a challenge to the fractionated wind hypothesis, especially since carbon is also in the same group as silicon, germanium, tin and lead, and should in principle feel the same radiative forces.

It is also worth noting that PG $1219+534$, one of our pulsators, shows measurable traces of silicon, despite being hotter than $32000 \mathrm{~K}$, while the spectroscopically similar stars Feige 66 and CD $-24^{\circ} 731$ do not. Could this be an indicator of the age of the sdB? If the star has a weak fractionated wind, as suggested by O'Toole (2004), then perhaps one of the differences between PG $1219+534$ and Feige $66 / C D-24^{\circ} 731$ is age. In order to determine this, however, diffusion models including silicon are required.

It seems clear that radiative acceleration is bringing heavy elements to the surface in these sdBs, and perhaps even expelling them through a stellar wind. We can at least qualitatively understand this in the case of the iron-group and heavier elements: in the hotter stars heavy elements are much more enhanced than in the cooler stars. We must also consider, however, that two of our "hot" stars are apparently single, while both of our "cool" stars are in close binary systems. Does this have any effect on the abundance patterns? It is unclear what the cause for the differences among the "hot" stars, Feige 66, CD $-24^{\circ} 731$, and PG $1219+534$, is, but binarity is one possibility. Our small number statistic is the main limiting factor for this discussion, so we must look forward to the abundance analyses of Edelmann et al. (2006), who have studied a larger number of binaries and single stars, albeit with optical spectra, which restricts the available heavier elements to iron and sometimes titanium.

\section{Summary and conclusions}

We have analysed high-resolution UV echelle spectra of five hot subdwarf B stars, two of which are member of the short-period, pulsating V361 Hya class. Abundances of no less than 25 elements including the iron group and even heavier elements such as tin and lead have been determined using LTE curve-of-growth and spectrum synthesis techniques. Our investigation was initiated to test the hypothesis that a correlation exists between the abundances of iron-group elements in sdB stars and pulsation. We have compared a hot pulsator (PG 1219+534) with a two non-pulsators with similar stellar parameters (Feige 66 and CD -24 731) and a cooler pulsator (Feige 48) with a similar non-pulsator (CPD $-64^{\circ} 481$ ), and found no consistent differences between the members of each pair. The heavy element abundance pattern of $\mathrm{CD}-24^{\circ} 731$ comes close to that observed for PG 1219+534 except for its low iron and nickel. Feige 66 has an even lower iron abundance, but its heavy metal abundance pattern does not match that of PG $1219+534$ at all. In other words the abundance patterns of two non-variable stars of similar temperature and gravity are too dissimilar for a conclusive comparison with a pulsator. This result leads us to suspect that there must be another, as yet unknown, discriminating factor between pulsating and non-pulsating sdB stars.

More generally, we have uncovered a potential solution to the discrepant effective temperatures from Balmer lines and helium ionisation equilibria, with a significant improvement found using supersolar metallicity models. Opacity sampling in place of distribution functions will sure lead to all model line profiles matching observations. Additionally, our spectra show light element abundance patterns typical for sdBs: carbon varies from virtually none to around 1 dex below solar, while nitrogen is within 0.5 dex of the solar value. There is evidence to support the fractionated weak stellar wind hypothesis of O'Toole (2004), as the heavy element abundances increase with temperature. We also find an interesting anti-correlation between the abundance of iron and the heavy element abundance relative to iron. More observations are needed to confirm this trend.

So somewhat frustratingly we must conclude that we cannot provide any insights into pulsation in sdB stars based on our spectroscopic measurements. On a more encouraging note, the results presented here will provide a valuable resource for theoretical work into diffusion in sdB stars. It will be important to study the diffusion of all iron group and heavier elements individually in order to explain the trend we have uncovered. We end then with a question and a challenge: how can we find the reason some sdB pulsate and some don't if not by spectroscopic means?

Acknowledgements. We thank the referee for constructive comments that have improved the manuscript. We would like to thank Michael Lemke for his support 
with the LINFOR code and Norbert Przybilla for useful discussions on the quality of atomic data. SJOT is supported by the Deutsches Zentrum für Luft- und Raumfahrt (DLR) through grant No. 50-OR-0202.

\section{References}

Baschek, B., Hoeflich, P., \& Scholz, M. 1982a, A\&A, 112, 76

Baschek, B., Scholz, M., Kudritzki, R. P., \& Simon, K. P. 1982b, A\&A, 108, 387

Behara, N. T. \& Jeffery, C. S. 2006, Baltic Astron., 15, 115

Charpinet, S., Fontaine, G., Brassard, P., et al. 1997, ApJ, 483, L123

Charpinet, S., Fontaine, G., Brassard, P., \& Dorman, B. 1996, ApJ, 471, L103

Charpinet, S., Fontaine, G., Brassard, P., Green, E. M., \& Chayer, P. 2005, A\&A 437, 575

Chayer, P., Fontaine, M., Fontaine, G., Wesemael, F., \& Dupuis, J. 2006, Baltic Astron., 15, 131

Edelmann, H., Heber, U., Altmann, M., Karl, C., \& Lisker, T. 2005, A\&A, 442, 1023

Edelmann, H., Heber, U., Napiwotzki, R. 2006, Baltic Astron., 15, 103

Edlén, B. 1966, Metrologia, 2, 71

Fontaine, G., \& Chayer, P. 1997, in The Third Conference on Faint Blue Stars, 169

Grevesse, N., \& Sauval, A. J. 1998, Space Sci. Rev., 85, 161

Han, Z., Podsiadlowski, P., Maxted, P. F. L., \& Marsh, T. R. 2003, MNRAS, 341, 669

Heber, U. 1986, A\&A, 155, 33

Heber, U., \& Edelmann, H. 2004, Ap\&SS, 291, 341

Heber, U., Hamann, W.-R., Hunger, K., et al. 1984a, A\&A, 136, 331
Heber, U., Hunger, K., Jonas, G., \& Kudritzki, R. P. 1984b, A\&A, 130, 119

Heber, U., Reid, I. N., \& Werner, K. 1999, A\&A, 348, L25

Heber, U., Reid, I. N., \& Werner, K. 2000, A\&A, 363, 198

Hirata, R., \& Horaguchi, T. 1995, VizieR On-line Data Catalog: VI/69

Kilkenny, D. 2002, in IAU Coll., 185, Radial and Nonradial Pulsations as Probes of Stellar Physics, ASP Conf. Ser., 259, 356

Kilkenny, D., Koen, C., O’Donoghue, D., \& Stobie, R. S. 1997, MNRAS, 285, 640

Koen, C., O’Donoghue, D., Pollacco, D. L., \& Nitta, A. 1998, MNRAS, 300, 1105

Koen, C., O’Donoghue, D., Pollacco, D. L., \& Charpinet, S. 1999, MNRAS, 305, 28

Lamontagne, R., Wesemael, F., Fontaine, G., \& Sion, E. M. 1985, ApJ, 299, 496 Lamontagne, R., Wesemael, F., \& Fontaine, G. 1987, ApJ, 318, 844

Maxted, P. F. L., Heber, U., Marsh, T. R., \& North, R. C. 2001, MNRAS, 326, 1391

Morton, D. C. 2000, ApJS, 130, 403

Morton, D. C. 2003, ApJS, 149, 205

O'Toole, S. J. 2004, A\&A, 423, L25

O'Toole, S. J., Heber, U., \& Benjamin, R. A. 2004, A\&A, 422, 1053

O'Toole, S. J., Jordan, S., Friedrich, S., \& Heber, U. 2005, A\&A, 437, 227

Przybilla, N., Nieva, M. F., \& Edelmann, H. 2006, Baltic Astron., 15, 107

Quirion, P.-O., Fontaine, G., \& Brassard, P. 2004, ApJ, 610, 436

Raassen, A. J. J., \& Uylings, P. H. M. 1997, A\&AS, 123, 147

Saffer, R. A., Bergeron, P., Koester, D., \& Liebert, J. 1994, ApJ, 432, 351

Unglaub, K. 2006, Baltic Astron., 15, 147

Uylings, P. H. M., \& Raassen, A. J. J. 1997, A\&AS, 125, 539 